\title{
Interplanar binding in graphite studied with the Englert-Schwinger equation
}

\author{
Angel Mã̃anes and Emilio Santos \\ Departamento de Fisica Teórica, Universidad de Santander, E-39005 Santander, Spain
}

(Received 7 October 1985)

\begin{abstract}
A model of a graphite crystal is used which consists of a set of parallel slabs of positive charge immersed in an electron sea. The density of electrons in the region between slabs is calculated from the Englert-Schwinger equation. That equation improves Thomas-Fermi theory by including exchange and inhomogeneity corrections to the kinetic energy. The results are in semiquantitative agreement with empirical data and are slightly better than previous calculations of the interplanar binding of graphite.
\end{abstract}

\section{INTRODUCTION}

In a series of papers, Schwinger and co-workers, ${ }^{1-6}$ have reconsidered the Thomas-Fermi theory, obtaining new equations that they have tested in atoms with a reasonable degree of success. This suggests the convenience of applying the equations to more complex electron systems, like molecules or solids, where other forms of Thomas-Fermi theory have been found useful. ${ }^{7,8}$

A system with convenient features for a test of Thomas-Fermi theory is graphite, in particular the study of the interplanar binding. Indeed, a number of papers have been devoted to the subject. 911 Graphite has the advantage, with respect to other systems, that it has two entirely different types of interactions. In the basal plane, the carbon atoms are held in a two-dimensional hexagonal lattice by strong covalent bonds, while the planes are held by much weaker energies which are neither covalent nor directional. The electrons binding the planes are most adequate for the Thomas-Fermi theory. Graphite is also convenient because many reliable experimental data exist. $^{10}$

We shall use a model of graphite that has been described elsewhere." It consists of a set of parallel "ionic slabs" of positive charge separated by regions with a slowly varying electron density. The "slabs" contain homogeneously distributed positive charges that simulate the carbon nuclei and a part of the electrons. In order to test the model, three different choices have been made (see Ref. 11 for details).

(1) Ionic slabs of width 2.36 a.u. (atomic units such that $\hbar=e=m=1$ are used throughout) containing the positive charges corresponding to the nuclei plus five electrons per atom (two electrons $1 s$ and three electrons in the sigma bonds). The electric field at the boundary of each slab is $\left|E_{0}\right|=0.6706$ a.u. We shall use Thomas-Fermi theory for the interslab regions that contain one electron per carbon atom.

(2) Homogeneous planar distributions of positive charge (i.e., slabs of zero width) corresponding to the charges of nuclei. The electric field at the layer is $\left|E_{0}\right|=4.024$ a.u. and Thomas-Fermi theory is applied to all electrons (i.e., six per carbon atom).

(3) Intermediate choice, having slabs of width 0.35 a.u., with charges corresponding to the nuclei plus two electrons $1 s$ per atom. The electric field at the slab surface is $\left|E_{0}\right|=2.6824$ a.u., and four electrons per carbon atom are put in the interslab regions.

This model of graphite is a kind of laboratory for the test of different approximations to Thomas-Fermi theory. Its main advantage is that the (partial nonlinear) differential equations of the theory can be solved analytically with the consequence that the various approximations can be tested in a clean way.

\section{THE EQUATION OF ENGLERT AND SCHWINGER}

Our starting point has been the equation derived by Englert and Schwinger ${ }^{4}$ in 1982 , which they applied to the calculation of the energy and the mean-square radii of atoms. We shall not be concerned with the equations derived later by the same authors, ${ }^{5,6}$ which are more accurate but lose the simplicity of the Thomas-Fermi theory. The Englert-Schwinger (ES) equation is (in atomic units)

$$
\nabla^{2} \bar{V}=-\frac{4}{3 \pi}\left\{\left[-2 \bar{V}+\left(\frac{11}{9 \pi}\right)^{2}\right]^{1 / 2}+\frac{11}{9 \pi}\right\}^{3},
$$

with

$$
\bar{V} \equiv V+\frac{1}{6 \pi}(-2 V)^{1 / 2},
$$

where $V(\mathbf{r})$ is the potential energy of an electron at $\mathbf{r}$ minus the Thomas-Fermi energy. A new, more rigorous, derivation of Eq. (1) has been given in Ref. 5(b), when the present calculation was already in progress. The function $\bar{V}$ involved in that derivation is slightly different from (2), so that the new equation would give different results for the interplanar binding of graphite. The modification, however, is likely to be not great, as discussed in the Appendix, but certainly this point should be tested by an actual calculation.

It can be realized that the ES equation (1) coincides with the Thomas-Fermi one in the limit $|V| \gg 1$. On the other hand, the ES equation has the same form as the Thomas-Fermi-Dirac (TFD) equation, but it has different numerical constants (e.g., $\frac{11}{9}$ instead of 1). The important point is that Eq. (1) contains corrections for inhomogenei- 
ty to the kinetic energy, which are not contained in the TFD equation. In fact, it has the same degree of accuracy as the Thomas-Fermi-Dirac-Kirzhnits equation (although it is simpler) as is shown in the following. ${ }^{12}$

We may start with the energy functional

$$
\begin{aligned}
E[n]=\int & {\left[\frac{1}{8 \pi}(\nabla V)^{2}+\frac{3}{10}\left(3 \pi^{2}\right)^{2 / 3} n^{5 / 3}\right.} \\
& \left.-\frac{3}{4}\left[\frac{3}{\pi}\right]^{1 / 3} n^{4 / 3}+\frac{1}{72} \frac{(\nabla n)^{2}}{n}\right] d^{3} r,
\end{aligned}
$$

where $n(\mathbf{r})$ is the number of electrons per unit volume and $-V(\mathbf{r})$ is the electrostatic potential. (Actually, we defined $V$ to be the potential energy of an electronwhich is minus the potential in atomic units-minus the Fermi energy.) The first term is the total Coulomb energy (it is infinite if the nuclei are considered pointlike, but this fact does not give rise to any problem, as we are interested only in energy differences), the second term is the usual Thomas-Fermi kinetic energy, the third term is the exchange energy in the form of Dirac, and the last one the inhomogeneity correction to the kinetic energy in the form of Kirzhnits. ${ }^{8}$ [Actually, Kirzhnits obtained an additional term, of the form $\nabla^{2} n$, which gives no contribution to the integral (3).]

The functional (3) is assumed stationary with respect to variations of $n$ and $V$ related through the Poisson equation

$$
\nabla^{2} V=4 \pi\left(n_{N}-n\right),
$$

where $n_{N}(\mathbf{r})$ is the nuclear charge density. The EulerLagrange equation corresponding to the minimization of (3), with the constraint that the total number of electrons is fixed, gives an integrodifferential equation. Putting $n$ from (4) in (3) leads to the Thomas-Fermi-DiracKirzhnits (TFDK) equation. This equation has been used previously ${ }^{11}$ for the calculation of the interlayer force in graphite, but a difficulty appeared due to the ambiguity in fixing the (four) boundary conditions needed for the solution of the differential equation. One of the purposes of the calculation reported in this paper is to test whether the ES equation is an adequate substitute for the TFDK equation.

In the derivation of the ES equation a pseudopotential $\bar{V}$ is introduced, see (2), and a pseudodensity $\widetilde{\rho}$ given by

$$
\widetilde{\rho} \equiv 3 \pi^{2} n-\frac{1}{8}\left(3 \pi^{2}\right)^{1 / 3} \nabla^{2}\left(n^{1 / 3}\right)
$$

so that the functional (3) can be rewritten in terms of $\tilde{\rho}$ and $\bar{V}$. This gives

$$
E=\frac{1}{3 \pi^{2}} \int\left[\frac{3}{10} \widetilde{\rho}^{5 / 3}-\frac{11}{12 \pi} \widetilde{\rho}^{4 / 3}+\frac{3 \pi}{8}(\nabla \bar{V})^{2}\right] d^{3} r,
$$

where the exchange contribution has been approximated ${ }^{4}$ by $\left(3 \pi^{2} n\right)^{4 / 3} \simeq \widetilde{\rho}^{4 / 3}$, i.e., we neglect corrections to the exchange correction.

The relations (2) and (5) are so chosen that the term containing the gradient of the density disappears from (6), and also the Poisson equation (4) is transformed into

$$
\nabla^{2} \bar{V}=-\frac{4}{3 \pi} \tilde{\rho} .
$$

(For the sake of simplicity we write only the expression valid outside the nuclei.) The functional (6) is stationary for variations of $\tilde{\rho}$ and $\bar{V}$ related through (7), which leads to (1). An equivalent equation, easily obtained from (1) and (7) is

$$
\widetilde{\rho}^{2 / 3}-\frac{22}{9 \pi} \widetilde{\rho}^{1 / 3}=-2 \bar{V} .
$$

Equations (7) and (8) and the functional (6) will be the basis for our calculation of the interlayer force of graphite.

From Eq. (8) we obtain that the condition

$$
\tilde{\rho}^{1 / 3} \geq \frac{11}{9 \pi}
$$

is necessary for $\widetilde{\rho}$, being a single valued function of $V$. Actually this is a consistency condition once Eqs. (7) and (8) are given, but our derivation of (8) implies [see (2)]

$$
2 \bar{V} \leq(6 \pi)^{-2} \simeq 0,
$$

which gives a constraint stronger than (9), namely

$$
\tilde{\rho}^{1 / 3} \geq \frac{22}{9 \pi} \text {. }
$$

A more detailed discussion about the alternative constraints (9) and (11) (in relation with atoms), can be seen in the original paper by Englert and Schwinger. ${ }^{4}$ We shall use the weakest form (9). In the version of the ES equation of Ref. 5(b) the same equation (8) is obtained so both constraints (9) and (11) remain as they stand. The changes in $\bar{V}$ will modify the energy, Eq. (6), and the boundary condition for $\bar{V}$, see Eq. (15) below, but these changes will likely not be very relevant for our problem, as discussed in the Appendix.

\section{CALCULATION OF THE ENERGY IN TERMS OF THE INTERLAYER DISTANCE}

As said in the Introduction, we consider a model of a graphite crystal consisting of a set of positively charged ionic slabs having width $2 Z_{0}$, separated by regions of width $R$ where the electron density is calculated by ES theory. We assume that the total energy is the sum of additive contributions associated with these two regions and that the energy of a slab is a constant when $R$ changes. Then, we put the slab energy equal to zero. The calculation follows closely that of Ref. 11 for the three choices of slab width mentioned in the Introduction. An essential point is that it is not necessary to work in full the solution of the ES, Eq. (1), in order to calculate the energy.

Instead of using Eq. (1), we start with Eqs. (17) and (18). In our problem, $\bar{V}$ and $\widetilde{\rho}$ are functions of a single coordinate $x$, this being perpendicular to the graphite layers. We choose $x=0$ at the surface of an ionic slab and find the solution from $x=0$ up to $x=R / 2$, i.e., to the plane halfway between the layers, the potential being symmetrical with respect to that plane. The boundary conditions for the potential are 


$$
\left.\frac{d V}{d x}\right|_{x=R / 2}=0,\left.\frac{d V}{d x}\right|_{x=0}=E_{0},
$$

$E_{0}$ being the electric field at the surface of the slab (see the Introduction for the value of $E_{0}$ ). Equation (2) allows finding the boundary conditions for $\bar{V}$ from (12). We start by differentiating Eq. (8) with respect to $x$, then replacing $d \tilde{\rho} / d x$ by $-(4 \tilde{\rho} / 3 \pi) d \tilde{\rho} / d \bar{V}^{\prime}$ [see (7)], and finally performing an integration of the resulting differential equation (with $\tilde{\rho}$ and $\bar{V}^{\prime}$ as variables) to get

$$
\bar{V}^{\prime}\left(\tilde{\rho}, C_{0}\right) \equiv \frac{d \bar{V}}{d x}=\left(\frac{8}{15 \pi} \tilde{\rho}^{5 / 3}-\frac{22}{27 \pi^{2}} \tilde{\rho}^{4 / 3}+C_{0}\right)^{1 / 2}
$$

where $C_{0}$ is a constant. Putting the first Eq. (12) into (13) we obtain the constant $C_{0}$ in terms of the density $\tilde{\rho}$ at $R / 2$ :

$$
C_{0}=\frac{22}{27 \pi^{2}} \widetilde{\rho}(R / 2)^{4 / 3}-\frac{8}{15 \pi} \widetilde{\rho}(R / 2)^{5 / 3} .
$$

The density at $x=0$ can be obtained from the second Eq. (12) and Eq. (2), which gives

$$
\bar{V}^{\prime}\left(\widetilde{\rho}(0), C_{0}\right)=E_{0}\left(1-\frac{1}{6 \pi}[-2 \bar{V}(0)]^{-1 / 2}\right) .
$$

Now $\bar{V}(0)$ and $\bar{V}^{\prime}(0)$ can be related to $\widetilde{\rho}(0)$ and $C_{0}$ through Eqs. (8) and (13), respectively. In this way we are able to obtain $\tilde{\rho}$ and $\bar{V}$ at $x=0$ and at $x=R / 2$ for each value of the constant $C_{0}$.

After that, we are in a position to calculate the interslab distance $R$, for each value of $C_{0}$, from (7) and (13). We get

$$
R=\frac{3 \pi}{2}\left(\frac{\bar{V}^{\prime}(0)}{\tilde{\rho}(0)}+\int_{\widetilde{\rho}(R / 2)}^{\widetilde{\rho}(0)} \frac{\bar{V}^{\prime}\left(\widetilde{\rho}, C_{0}\right)}{\tilde{\rho}^{2}} d \widetilde{\rho}\right] .
$$

The possible values of $C_{0}$ are constrained by those of $\tilde{\rho}(R / 2)$ [see (14)], and these by the conditions (9) and (11) already discussed.

The constraint (9) gives rise to a maximum possible value for $R$, which we label $R_{M}$. If the slabs are separated by a distance greater than $R_{M}$, the density becomes discontinuous, a fact similar to the one appearing in the Thomas-Fermi-Dirac theory of the atom. The values of $R_{M}$ obtained for the three different models described in the Introduction are given in the first column of Table I. They are greater than the empirical equilibrium value 6.3 a.u., which is a necessary condition if we want a theoretical prediction for this equilibrium distance. The calculation of the energy is straightforward. Starting from (6) we obtain the energy per carbon atom associated to the electron in the interslab region to be

$$
E(R)=\frac{A}{144 \pi}\left[-C_{0} R+\left(10 \widetilde{\rho}(0)^{2 / 3}-26 \times \frac{11}{9 \pi} \widetilde{\rho}(0)^{1 / 3}\right] \bar{V}^{\prime}(0)+\frac{22}{9 \pi} \int_{\tilde{\rho}(R / 2)}^{\tilde{\rho}(0)} \bar{V}^{\prime}\left(\widetilde{\rho}, C_{0}\right) \widetilde{\rho}^{-2 / 3} d \widetilde{\rho}\right]
$$

where $A=18.735$ a.u., is the area corresponding to a carbon atom (see Ref. 11 for details). In practice, we have used a computer program such that, for each value of $C_{0}$, we get the interslab distance $R$ and the energy per atom E.

The results for the energy $E(R)$ with model II are shown in Fig. 1. The theory predicts binding with the equilibrium distance at $R_{m}=7.23$ a.u., a value that is far away from the experimental one and also greater than the value obtained, with the same model, in Ref. 9, where the inhomogeneity correction to the kinetic energy was included as a perturbation but it was not in the minimization of the functional.
Taking the zero of energy at the maximum interlayer separation we obtain, as a first estimate for the exfoliation energy, the value of the energy at the minimum, $0.4 \times 10^{-4}$ a.u., to be compared with the experimental value $0.84 \times 10^{-3}$ a.u. In consequence, we arrive at the conclusion that the quantum corrections do not produce enough binding between the layers. (However, the method for the calculation of the binding energy is somewhat ambiguous.) In Table I the corresponding values for the other two models are also shown.

To make easier the comparison of our values with the empirical values and with other theoretical work, we have fitted to our results for the energy the following Morse

TABLE I. Calculated values of (1) the maximum interslab distance $R_{M}$, (2) two equilibrium distances [for $E(R)$ and for $E(R)+E_{c}(R)$ ] $R_{m}$, and (3) the energy without correlation energy included [E(R)], and with it $\left[E(R)+E_{c}(R)\right]$ at this distance $R_{m}$ (all in atomic units). The experimental equilibrium distance is 6.33 a.u.

\begin{tabular}{cccccc}
\hline \hline Graphite & $R_{M}$ & $R_{m}$ & $\begin{array}{c}E\left(R_{m}\right) \\
\left(10^{-3} \text { a.u. }\right)\end{array}$ & $R_{m}$ & $\begin{array}{c}E\left(R_{m}\right)+E_{c}\left(R_{m}\right) \\
\left(10^{-3} \text { a.u. }\right)\end{array}$ \\
\hline Model 1 $\left(1 e^{-/ \text {atom })}\right.$ & 7.776 & 7.36 & -0.0366 & 6.64 & -0.358 \\
Model 2 $\left(6 e^{-/ \text {atom })}\right.$ & 7.658 & 7.23 & -0.0398 & 6.45 & -0.403 \\
Model 3 $\left(4 e^{- \text {/atom) }}\right.$ & 7.547 & 7.12 & -0.0395 & 6.34 & -0.397 \\
\hline \hline
\end{tabular}


curve:

$$
E(R)=a_{1}\left(e^{-a_{2}\left(R-a_{3}\right)}-1\right)^{2} .
$$

This curve for the total energy as a function of the interlayer distance is of the scaled form discussed by several authors in connection with the existence of a universal binding-energy-distance relation for metals, molecules, and other systems. ${ }^{13}$ The parameters of the fit are given in the first row of Table II, as well as the parameters corresponding to the experimental values and to other calculations. For the sake of comparison with previous works $^{9,11}$ we have also fitted an unscaled curve to the energy

$$
E(R)=-A_{0} R^{-4}+B e^{-\alpha R}+C .
$$

The values of the parameters for the best fit are given in the first row of Table III.

We see that, with the extrapolation procedure used, the results from our calculation of the energy do not give a clear improvement of the values obtained in Ref. 10, where other terms were included in the energy functional, the most important one being the correlation energy. In fact, we are not able to reproduce the right position of the equilibrium distance, and we obtain a too high value for the compressibility. Anyway as the cutoff for the model (maximum interlayer distance) is very near the binding region, we think that the extrapolation of the data by the use of any prescribed function is highly doubtful. As we will see later in Sec. IV the corrections due to the correlation energy greatly improve these results.

From the Poisson equation (7) and using (13) it is possible to obtain a relation between the density $\tilde{\rho}(x)$ and the distance $x$ from the slab. This relation in integral form is

$$
\begin{gathered}
x=\frac{3 \pi}{4}\left[\bar{V}^{\prime}\left(\tilde{\rho}(0), C_{0}\right) \frac{1}{\tilde{\rho}(0)}-\bar{V}^{\prime}\left(\tilde{\rho}(x), C_{0}\right) \frac{1}{\tilde{\rho}(x)}\right. \\
\left.+\int_{\tilde{\rho}(x)}^{\tilde{\rho}(0)} \bar{V}^{\prime}\left(\tilde{\rho}, C_{0}\right) \frac{1}{\tilde{\rho}^{2}} d \tilde{\rho}\right],
\end{gathered}
$$

where

$$
\tilde{\rho}(x) \in[\tilde{\rho}(R / 2), \tilde{\rho}(0)]
$$

for each interslab distance $R$. In order to obtain $n(x)$ it is

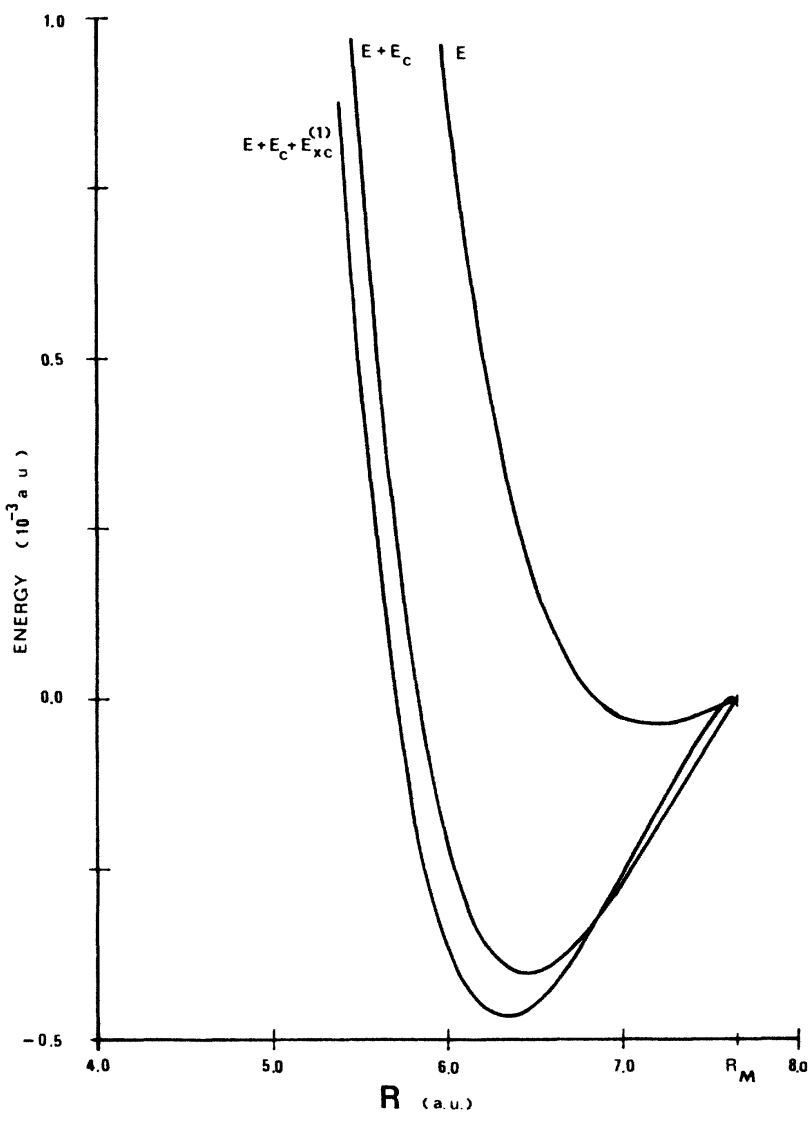

FIG. 1. Calculation of the energy for graphite model 2. $E$ is the Engelert-Schwinger energy given by (17) and referred to its value at $R_{M}$. We include the correlation energy, given by (22), in $E+E_{c}$. The value at $R_{M}, E\left(R_{M}\right)+E_{c}\left(R_{M}\right)$, is taken as zero of energy for the $E+E_{c}$ curve. The third curve, $E+E_{c}+E_{\mathrm{xc}}^{(1)}$, includes gradient corrections to the exchange and correlation energies, given by (28); it is also referred to its value at $R_{M}$.

necessary to invert Eq. (5), and we obtain

$$
n(x)=\frac{1}{3 \pi^{2}}\left[\tilde{\rho}(x)+\frac{1}{8} \frac{d^{2} \tilde{\rho}^{1 / 3}}{d x^{2}}\right] .
$$

The second derivative of $\tilde{\rho}^{1 / 3}$ is readily obtained from (7)

TABLE II. Morse-potential parameters and structural constants for graphite model 2, with three different prescriptions for the calculation of the energy. The last two rows correspond to the calculated and experimental values given in Ref. 10 . The force constant, $K=\left[d^{2} E / d R^{2}\right]_{R_{m}}$, and the compressibility $\kappa_{c}=A\left(2 R_{m} K\right)^{-1}$, are obtained from the fitting. This fit is performed between $R=5.4$ and $R_{M}$ for $E$ and between $R=4.3$ and $R_{M}$ in the other two cases.

\begin{tabular}{lccccc}
\hline & $\begin{array}{c}\text { Binding energy } a_{1} \\
\left(10^{-3} \text { a.u./C-atom) }\right.\end{array}$ & $\begin{array}{c}a_{2} \\
\left(\text { a.u. }{ }^{-1}\right)\end{array}$ & $\begin{array}{c}\text { Equilibrium } \\
\text { distance } a_{3} \\
(\text { a.u. })\end{array}$ & $\begin{array}{c}\text { Compressibility } \kappa_{c} \\
\left(10^{-12} \mathrm{~cm}^{2} / \text { dyn }\right)\end{array}$ & $\begin{array}{c}\text { Force constant } K \\
\left(10^{-2} \text { a.u. }\right)\end{array}$ \\
\hline$E$ & 0.62 & 0.66 & 7.2 & 8.03 & 0.054 \\
$E+E_{c}$ & 1.44 & 0.666 & 6.47 & 3.83 & 0.13 \\
$E+E_{c}+E_{x c}^{(1)}$ & 1.26 & 0.696 & 6.44 & 4.05 & 0.122 \\
Ref. 10 & 4.03 & 0.866 & 5.27 & 0.97 & 0.63 \\
Expt. (Ref. 10) & 0.84 & 1.028 & 6.33 & 2.7 & 0.19 \\
\hline \hline
\end{tabular}




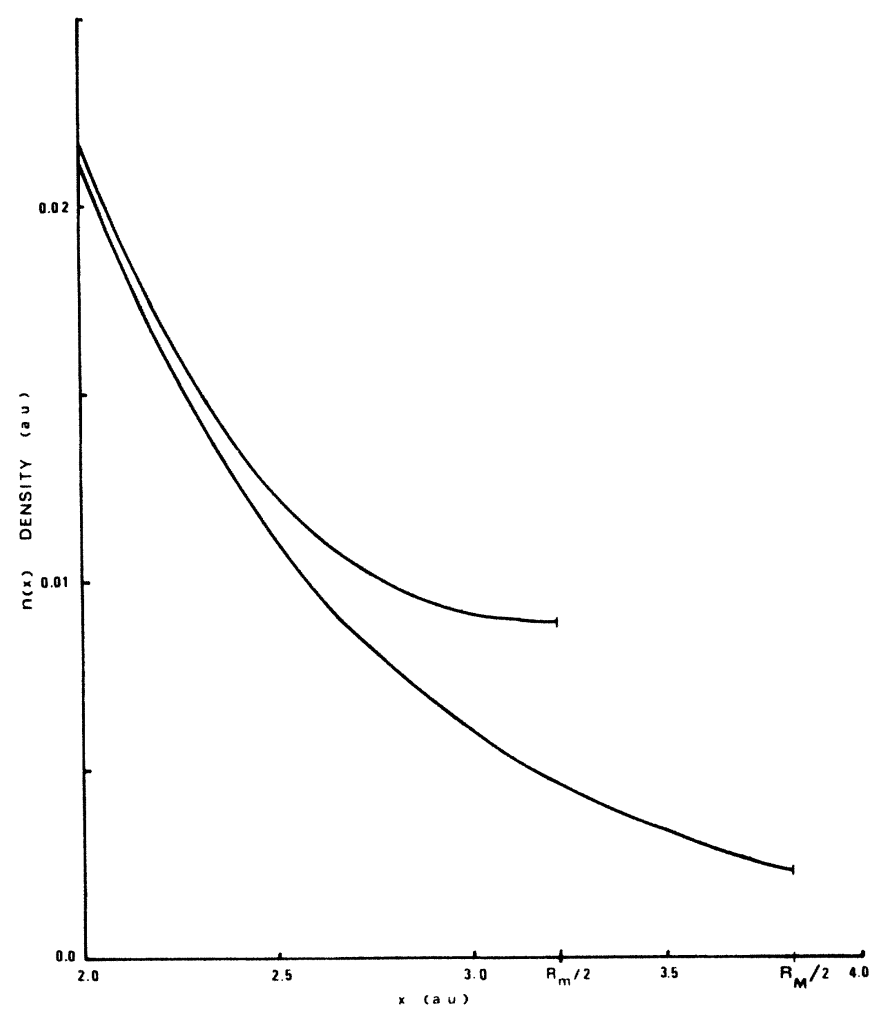

FIG. 2. Electron density in the interslabs region for graphite model 2 from the Englert-Schwinger equation, as a function of the distance to the nearest layer. It is shown for the maximum interlayer distance $\boldsymbol{R}_{M}=7.658$ a.u. (lower line) and for the calculated equilibrium distance $R_{m}=6.45$ a.u. (upper line).

and (13) in terms of $\widetilde{\rho}(x)$ as will be discussed in Sec. V. The results for $n(x)$ are given in Fig. 2 for two different values of the interslab distance.

\section{INFLUENCE OF THE CORRELATION ENERGY}

As is discussed in Sec. II and in Ref. 12, further terms of the energy density functional (1) are lacking in the ES equation. The most important one is the correlation energy. We have used the following functional proposed by Gunnarsson and Lundqvist in the LDA as is quoted in Ref. 14:

$$
E_{c}[n]=\int \epsilon_{c}(n) n d^{3} r,
$$

with

$$
\epsilon_{c}(n)=-\frac{C_{1}}{2}\left[\left(1+S^{3}\right) \ln \left(1+\frac{1}{S}\right)-S^{2}+\frac{S}{2}-\frac{1}{3}\right] .
$$

Here

$$
S=\frac{1}{C_{2}}\left(\frac{3}{4 \pi}\right)^{1 / 3} n(\mathbf{r})^{-1 / 3}
$$

and the numerical values of the parameters are $C_{1}=0.0666$ and $C_{2}=11.4$ in atomic units. The function-

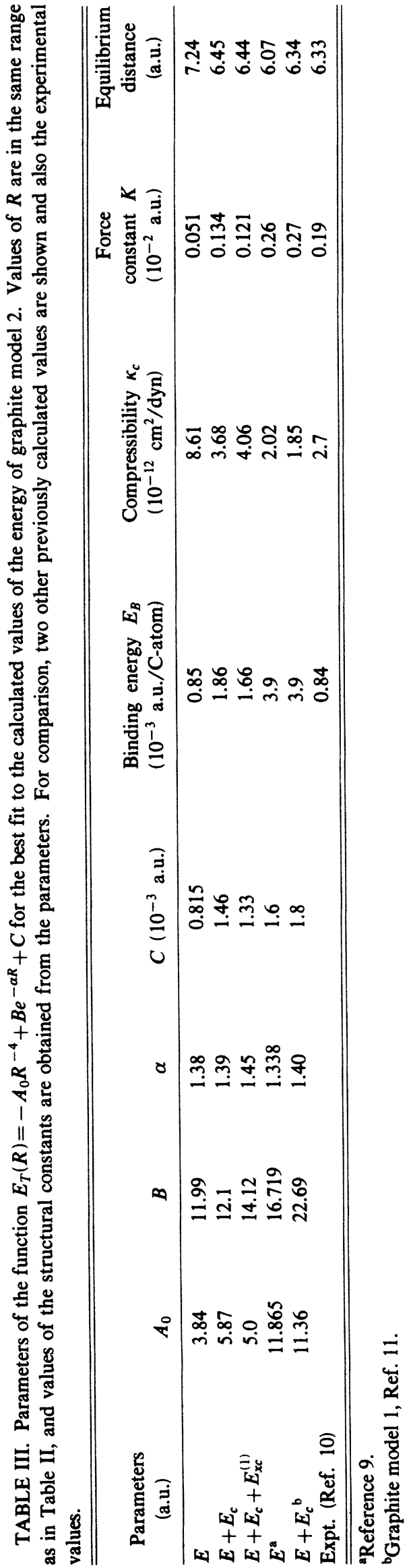


al form of $E_{c}$ is the same as used in Ref. 10, where slightly different values of $C_{1}$ and $C_{2}$ were taken. In our case, taking Poisson equation into account, we get

$$
\begin{aligned}
& E_{c}(R)=\frac{3 \pi}{4} A\left[\epsilon_{c}(\widetilde{\rho}(0)) \bar{V}^{\prime}\left(\widetilde{\rho}(0), C_{0}\right)\right. \\
&\left.-\int_{\widetilde{\rho}(R / 2)}^{\widetilde{\rho}(0)} \bar{V}^{\prime}\left(\widetilde{\rho}, C_{0}\right) \frac{d \epsilon_{c}}{d \widetilde{\rho}} d \widetilde{\rho}\right],
\end{aligned}
$$

where use has been made of the boundary condition $\bar{V}^{\prime}\left(\widetilde{\rho}(R / 2), C_{0}\right)=0$, the first of Eqs. (12).

In Fig. 1 the results are given for $E(R)+E_{c}(R)$. The values for the parameters of the fitted curves (18) and (19) are given in the second row of Tables II and III, respectively. In Fig. 3 the correlation energy $E_{c}(R)$ is shown together with other corrections to be discussed later on in Sec. V. The correctness of our perturbative treatment is confirmed by the values of the different contributions taken at their maximum interlayer distance $R$, namely

$$
\begin{aligned}
& E\left(R_{M}\right)=6.775925 \text { a.u. , } \\
& E_{c}\left(R_{M}\right)=-0.421776 \text { a.u. },
\end{aligned}
$$

i.e., $E_{c}$ only about $6 \%$ of $E$.

The agreement with the experimental values for the curve given by Eq. (18) is quite good: $3 \%$ deviation for the equilibrium distance and $36 \%$ and $42 \%$ for the binding energy and the compressibility, respectively, everything by excess. The parameter $a_{2}$, which gives the decay length of the total energy towards zero, is reproduced better by the calculation of DiVincenzo et al. ${ }^{10}$ where a continuous exponentially decaying density is used. As we have mentioned, this is one of the shortcomings of the present model. For the curve (19) and with our definition of the binding energy, i.e.,

$$
E_{B}=E_{T}(\infty)-E_{T}\left(R_{m}\right),
$$

where $R_{m}$ is the equilibrium distance, we obtain a value that is different from the one obtained with (18), due to the constant $C$ introduced in the former. ${ }^{9}$ In Table III several other calculations taken from Ref. 11 are also shown.

\section{HIGHER-ORDER CORRECTIONS}

As an estimation of the accuracy of the calculations made, we have studied several higher corrections to the energy functional (1). We have calculated also some of the contributions which were disregarded in going from Eq. (3) to Eq. (6) by means of the relations (2) and (5).

\section{A. Inhomogeneity correction to exchange and correlation}

The first contribution that we have considered is the gradient corrections to exchange and correlation energies

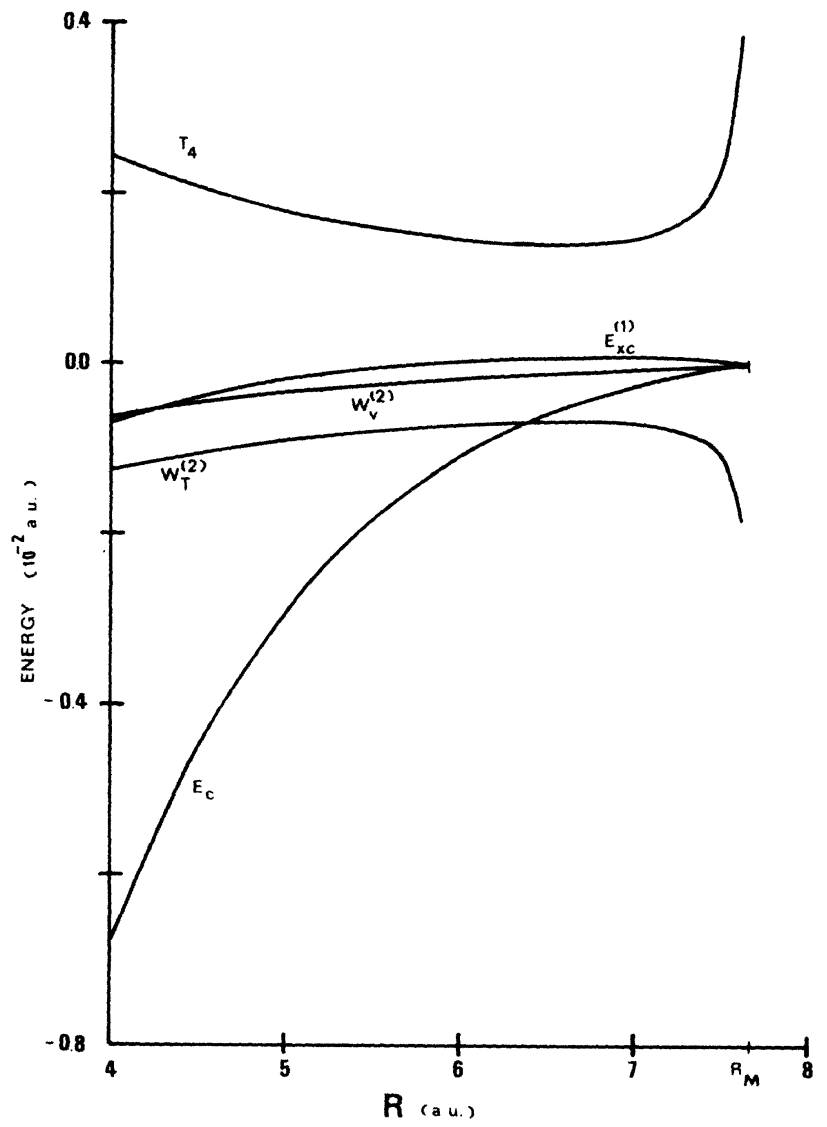

FIG. 3. Corrections to the energy of graphite model 2 as a function of the interlayer separation. Included are the correlation energy, $E_{c}$, Eq. (25); the first gradient correction to the exchange and correlation energies, $E_{\mathrm{xc}}^{(1)}$, Eq. (28); the fourth-order correction to the kinetic energy, $T_{4}$, Eqs. (38) and (39); the second inhomogeneity correction to the Coulomb energy, $W_{V}^{(2)}$, Eq. (47); and the second-order inhomogeneity corrections to the Thomas-Fermi and Kirzhnits kinetic energies, $W_{T}^{(2)}$ [i.e., the sum of the second part of (44) and (46)]. The zero of energy is taken at $R_{M}$.

in the form given by Langreth and Mehl: ${ }^{15}$

$$
\begin{aligned}
E_{\mathrm{xc}}^{(1)}= & -\frac{7}{9} B_{1} \int \frac{(\nabla n)^{2}}{n^{4 / 3}} d^{3} r \\
& +2 B_{1} \int \frac{(\nabla n)^{2}}{n^{4 / 3}} e^{-B_{2}|\nabla n| / n^{7 / 6}} d^{3} r,
\end{aligned}
$$

where $B_{1}=2.14 \times 10^{-3}$ a.u. and $B_{2}=0.262$ a.u. It is a first gradient correction, $E_{\mathrm{xc}}^{(1)}$, as the inhomogeneity correction to the kinetic energy. For our systems this expression becomes:

$$
E_{\mathrm{xc}}^{(1)}(R)=\frac{A B_{1}}{\left(3 \pi^{2}\right)^{2 / 3}} \int_{\widetilde{\rho}(R / 2)}^{\widetilde{\rho}(0)}\left(-\frac{7}{9}+2 e^{-B_{2} X(\widetilde{\rho})\left(3 \pi^{2} \widetilde{\rho}\right)^{1 / 6}}\right) X(\widetilde{\rho}) d \widetilde{\rho},
$$


where

$$
X(\widetilde{\rho}) \equiv-\frac{d \widetilde{\rho}}{d x} \tilde{\rho}^{-4 / 3}=3 \bar{V}^{\prime}\left(\widetilde{\rho}, C_{0}\right) \tilde{\rho}^{-2 / 3}\left(\tilde{\rho}^{1 / 3}-\frac{11}{9 \pi}\right)^{-1}
$$

and Eqs. (8) and (13) have been used in (29).

Equation (28) is obtained from (27) by means of the definition of $\tilde{\rho}$ in (5) and retaining only the terms of highest order. The results for $E_{\mathrm{xc}}^{(1)}(R)$ are given in Fig. 3 as a function of the interlayer distance. The value at $R_{M}$ is $E_{\mathrm{xc}}^{(1)}(R)=0.02068$ a.u., i.e., only $0.3 \%$ of $E$ and $5 \%$ of $E_{c}$. It is seen from the general trend that $E_{\mathrm{xc}}^{(1)}$ appears as a small antibonding effect in contrast with the monotonous increase shown in the calculations of Ref. 10 (see Fig. 1 there). The results of the fit of curve (18) to the total energy, $E+E_{c}+E_{\mathrm{xc}}^{(1)}$, are given in the third row of Table II. A slight improvement of the binding energy $\left(a_{1}\right)$ and the equilibrium distance $\left(a_{3}\right)$ is obtained. However, the compressibility goes in the wrong direction as far as it is mainly dominated by the decaying length $\left(a_{2}\right)$, which is yet too much separated from the experimental value.

\section{B. Second inhomogeneity correction to the kinetic energy} ed:

The fourth-order correction for inhomogeneity to the kinetic energy as given by Hodges ${ }^{16}$ is the next correction includ-

$$
E_{T}^{(4)}[n]=\frac{1}{540\left(3 \pi^{2}\right)^{2 / 3}} \int n^{1 / 3}\left[\left(\frac{\nabla^{2} n}{n}\right)^{2}-\frac{9}{8} \frac{\nabla^{2} n}{n}\left[\frac{\nabla n}{n}\right)^{2}+\frac{1}{3}\left(\frac{\nabla n}{n}\right)^{4}\right] d^{3} r
$$

Another problem of the present model appears here, namely the second derivative of the density $\widetilde{\rho}$ at half the interlayer distance diverges when we approach the maximum separation between layers, $R_{M}$. There is also a discontinuity of the first kind in the first derivative of $\tilde{\rho}(x)$, as is shown in the analysis that follows.

From (8) and (13) using $\widetilde{\rho}^{1 / 3}$ for simplicity, we obtain

$$
\frac{d \tilde{\rho}^{1 / 3}}{d x}=-\bar{V}^{\prime}\left(\tilde{\rho}, C_{0}\right)\left(\tilde{\rho}^{1 / 3}-\frac{11}{9 \pi}\right)^{-1},
$$

and

$$
\frac{d^{2} \tilde{\rho}^{1 / 3}}{d x^{2}}=\frac{4}{3 \pi} \frac{\tilde{\rho}}{\tilde{\rho}^{1 / 3}-\lambda}-\frac{\left[\bar{V}^{\prime}\left(\tilde{\rho}, C_{0}\right)\right]^{2}}{\left(\tilde{\rho}^{1 / 3}-\lambda\right)^{3}}
$$

Now, we separate the two possibilities for the minimal value of the density $\tilde{\rho}$ at half the layer distance obtained from (9): (a)

$$
\tilde{\rho}^{1 / 3}\left(R_{M} / 2\right)=11 / 9 \pi
$$

and (b)

$$
\tilde{\rho}^{1 / 3}(R / 2)>11 / 9 \pi
$$

In case (a) we have from (14)

$$
C_{0}\left(R_{M}\right)=\frac{2}{15 \pi} \lambda^{5}, \lambda \equiv \frac{11}{9 \pi},
$$

so equation (13) becomes

$$
\begin{aligned}
\bar{V}^{\prime}\left(\widetilde{\rho}, C_{0}\right)=\left(\frac{2}{3 \pi}\right)^{1 / 2}[ & 2 \lambda^{3}(z-\lambda)^{2}+4 \lambda^{2}(z-\lambda)^{3} \\
& \left.+3 \lambda(z-\lambda)^{4}+\frac{4}{5}(z-\lambda)^{5}\right]^{1 / 2},
\end{aligned}
$$

where $z \equiv \tilde{\rho}^{1 / 3}$, and finally from (31) and (32), we get

$$
\begin{aligned}
& \left.\frac{d \tilde{\rho}^{1 / 3}}{d x}\right|_{x=R_{M} / 2}=-\frac{2}{\sqrt{3 \pi}} \lambda^{3 / 2}, \\
& \left.\frac{d^{2} \widetilde{\rho}^{1 / 3}}{d x^{2}}\right|_{x=R_{M} / 2}=\frac{4}{3 \pi} \lambda^{2} .
\end{aligned}
$$

For the case (b) and using (12) in (31) and (32) we obtain

$$
\begin{aligned}
& \left.\frac{d \tilde{\rho}^{1 / 3}}{d x}\right|_{x=R_{M} / 2}=0, \\
& \left.\frac{d^{2} \widetilde{\rho}^{1 / 3}}{d x^{2}}\right|_{x=R_{M} / 2}=\frac{4}{3 \pi} \frac{\tilde{\rho}(R / 2)}{\tilde{\rho}(R / 2)^{1 / 3}-\lambda} .
\end{aligned}
$$

The second Eq. (35) shows the divergence mentioned before, as $R$ gets closer to its maximum value $R_{M}$, i.e., $\tilde{\rho}^{1 / 3}(R / 2) \rightarrow \lambda$. From (35) we also see that $\left[d^{2} \widetilde{\rho} / d x^{2}\right]_{R / 2}$ has its minimum value at $\tilde{\rho}^{1 / 3}(R / 2)=\frac{5}{4} \lambda$, i.e., $C_{0}(R)=0$ and $R \simeq 7.23$ a.u., the equilibrium value for $E$, so the condition

$$
\tilde{\rho}^{1 / 3} \geq \frac{5}{4} \frac{11}{9 \pi}
$$

will be better than (9) as far as a monotonous behavior of $\left[\tilde{\rho}^{\prime \prime}\right]_{R / 2}$ as a function of $R$ is desirable, and in order to eliminate the divergence of the $E_{T}^{(4)}$ correction. Anyway, it must be remembered that, using Eq. (36), we remain yet outside the range, given by (11), for the validity of the equations, and that it is not possible to obtain binding from $E(R)$ with (36).

The following expression is obtained for the correction at $R$ :

$$
\begin{aligned}
T_{4}(R) \equiv E_{T}^{(4)}(R)=\frac{A}{240 \pi} & \bar{V}^{\prime}\left(\tilde{\rho}(0), C_{0}\right) I(\tilde{\rho}(0)) \\
& \left.-\int_{\tilde{\rho}(R / 2)}^{\widetilde{\rho}(0)} \bar{V}^{\prime}\left(\widetilde{\rho}, C_{0}\right) \frac{d I}{d \tilde{\rho}} d \widetilde{\rho}\right),
\end{aligned}
$$


where $I(\widetilde{\rho})$ is given by

$$
\begin{aligned}
I(\widetilde{\rho})= & \left\{\left[\left(\widetilde{\rho}^{1 / 3}\right)^{\prime \prime} \widetilde{\rho}^{-2 / 3}\right]^{2}+\frac{5}{8}\left(\widetilde{\rho}^{1 / 3}\right)^{\prime \prime}\left[\left(\widetilde{\rho}^{1 / 3}\right)^{\prime}\right]^{2} \widetilde{\rho}^{-5 / 3}\right. \\
& \left.+\frac{1}{4}\left[\left(\widetilde{\rho}^{1 / 3}\right)^{\prime}\right]^{4} \widetilde{\rho}^{-2}\right\},
\end{aligned}
$$

and relations (13), (31), and (32) are used to obtain the derivatives of $\bar{V}$ and $\widetilde{\rho}^{1 / 3}$ in terms of $\widetilde{\rho}$. The results are given in Fig. 3. The value for the correction at $R_{M}$ is

$$
T_{4}\left(R_{M}\right)=0.012768663 \text { a.u. , }
$$

although it diverges when $R \rightarrow R_{M}$, as we have discussed.

\section{Terms neglected in the derivation of the Englert-Schwinger equation}

The other terms that we want to mention do not suppose new parts in the energy functional (3), but they arise from the equation (3) when the more accurate relations

$$
V \simeq \bar{V}+\frac{1}{6 \pi}(-2 \bar{V})^{1 / 2}-\frac{1}{36 \pi^{2}}
$$

and

$$
3 \pi^{2} n \simeq \widetilde{\rho}+\frac{1}{8} \Delta \widetilde{\rho}^{1 / 3}+\frac{1}{192} \Delta\left(\frac{\Delta \widetilde{\rho}^{1 / 3}}{\widetilde{\rho}^{2 / 3}}\right),
$$

obtained from (2) and (5), are used for $n$ and $V$. All other contributions of the same order have been included in (6) except the one which is obtained from the exchange energy term. It is given by

$$
W_{x}^{(1)}=-\frac{1}{24 \pi^{3}} \int \tilde{\rho}^{1 / 3} \nabla^{2} \widetilde{\rho}^{1 / 3} d^{3} r,
$$

and it is of the same order of magnitude as the first part of $E_{\mathrm{xc}}^{(1)}$ in (27), as is shown by its expression in the graphite system:

$$
\begin{aligned}
W_{x}^{(1)}(R)= & \frac{A}{216 \pi^{3}} \int_{\widetilde{\rho}(R / 2)}^{\widetilde{\rho}(0)} X(\widetilde{\rho}) d \widetilde{\rho} \\
& -\frac{A}{72 \pi^{3}}\left[-\widetilde{\rho}^{\prime}(0)\right] \widetilde{\rho}(0)^{-1 / 3},
\end{aligned}
$$

where $X(\widetilde{\rho})$ is given by (29).

It is straightforward to obtain the contributions of higher order from the four terms in (3). The results are

$$
\begin{aligned}
& W_{T 0}^{(2)}=\frac{1}{1152 \pi^{2}} \int\left[K \Delta\left(\frac{\Delta K}{K^{2}}\right)+\left(\frac{\Delta K}{K}\right)^{2}\right] K d^{3} r, \\
& W_{x}^{(2)}=-\frac{1}{576 \pi^{3}} \int\left[K \Delta\left(\frac{\Delta K}{K^{2}}\right)+\frac{1}{2}\left(\frac{\Delta K}{K}\right)^{2}\right] d^{3} r, \\
& W_{T 2}^{(2)}=\frac{1}{864 \pi^{2}} \int\left[\nabla K \nabla\left(\frac{\Delta K}{K}\right)+\frac{1}{2}\left(\frac{\nabla K}{K}\right)^{2} \Delta K\right] d^{3} r,
\end{aligned}
$$

and

$$
W_{V}^{(2)}=\frac{1}{2592 \pi^{3}} \int(\nabla K)^{2} d^{3} r
$$

where $K=K(\mathbf{r}) \equiv \widetilde{\rho}(\mathbf{r})^{1 / 3}$. Only $W_{T 2}^{(2)}$ and the second term in (44) have been calculated, and their sum is shown in Fig. 3. These corrections to the kinetic energy present the same behavior as the $T_{4}$ contribution at the maximum interslab distance $R_{M}$, i.e., they diverge. A complete analysis of these two parts and of $W_{x}^{(2)}$ would be necessary in order to see whether some desirable cancellations with the $T_{4}$ part occur. The last term, $W_{V}^{(2)}$, is shown also in Fig. 2. Its value at $R_{M}$ is $W_{V}^{(2)}\left(R_{M}\right)$ $=0.3997 \times 10^{-2}$ a.u., even smaller than $T_{4}\left(R_{M}\right)$.

As a conclusion of this section, we may confidently state that amongst all corrections to the EnglertSchwinger theory, only the correlation gives a very important contribution to binding. Other corrections are small in absolute value and also give small contribution to binding or antibonding. Some of the contributions diverge when we approach the region where the basic theory becomes invalid.

\section{ACKNOWLEDGMENT}

We acknowledge financial support by the Comisión Asesora de Investigación Científica y Técnica (Madrid, Spain).

\section{APPENDIX}

In the following we make an estimation of the changes which may be produced in the results of our calculation by changing the Engelert-Schwinger equations (1) and (2) to those of Ref. 5(b).

First of all, the relation (8) is not modified by the definition of $\bar{V}$ given in Eq. (174) of Ref. 5(b), i.e.,

$$
\begin{aligned}
\bar{V}= & (V+\zeta)+\frac{7}{6 \pi}[2(-V-\zeta)]^{1 / 2} \\
& +\frac{1}{2}\left[\left(\frac{11}{9 \pi}\right)^{2}-\left(\frac{7}{6 \pi}\right)^{2}\right] .
\end{aligned}
$$

In consequence, the constraints (9) and (11) remain as they stand. In fact if we redefine $(-4 \pi / 3) \widetilde{\rho}$ as the right-hand side of Eq. (1) [Eq. (176) of Ref. 5(b)], we obtain, from Eq. (175) of Ref. 5(b),

$$
[2(-V-\zeta)]^{1 / 2}=\widetilde{\rho}^{1 / 3}-\frac{1}{18 \pi},
$$

and then the relation between $\widetilde{n}=3 \pi^{2} n$ and $\widetilde{\rho}$ is, from Eq. (170) of Ref. 5(b),

$$
\widetilde{n}=\widetilde{\rho}+\frac{1}{8} \Delta \widetilde{\rho}^{1 / 3}+\frac{1}{1458 \pi^{2}}-\frac{1}{108 \pi^{2}} \widetilde{\rho}^{1 / 3},
$$

whose last two terms are negligible, so the equation (A3) is identical to (5), and both definitions of $\tilde{\rho}$ are equivalent. From (A2) and (A1) we obtain finally

$$
\widetilde{\rho}^{2 / 3}-\frac{22}{9 \pi} \widetilde{\rho}^{1 / 3}=-2 \bar{V},
$$

i.e., Eq. (8).

There are two changes to be considered in our paper if the new definition (A1) of $\bar{V}$ is used: The first one is in the energy functional equation (6), where the numerical 
factor $11 / 12 \pi$ in front of $\tilde{\rho}^{4 / 3}$ should be changed to $23 / 12 \pi$. The second one concerns the boundary condition (15), where $1 / 6 \pi$ should be changed to $7 / 6 \pi$. The influence of this change in the values of $\bar{V}^{\prime}\left(\tilde{\rho}(0), C_{0}\right)$ is only about $15 \%$, because the potential $\bar{V}(x)$ takes its biggest value at $x=0$.

As far as the relation between $\bar{V}$ and $\widetilde{\rho}$ is always given by Eq. (8), Eq. (13) for $\bar{V}^{\prime}\left(\widetilde{\rho}, C_{0}\right)$ is unchanged. Then, the influence of the redefinition of $\bar{V}$ in the calculated values of the interlayer distance $R$ [Eq. (16)] and the energy $E(R)$ [Eq. (17)] is only due to change in the value of the term $\bar{V}^{\prime}\left(\widetilde{\rho}(0), C_{0}\right)$. For $R$ the change is only about $2 \%$, and it is about $16 \%$ in the values of $E(R)$. In any case, we are only interested in energy differences, $E(R)-E\left(R_{M}\right)$, so the influence is even smaller because the values of $\tilde{\rho}$ at $x=0$ are practically $R$ independent.
${ }^{1}$ J. Schwinger, Phys. Rev. A 22, 1827 (1980).

2J. Schwinger, Phys. Rev. A 24, 2353 (1981).

${ }^{3}$ L. L. de Raad, Jr. and J. Schwinger, Phys. Rev. A 25, 2399 (1982).

${ }^{4}$ B. G. Englert and J. Schwinger, Phys. Rev. A 26, 2322 (1982).

5(a) B. G. Englert and J. Schwinger, Phys. Rev. A 29, 2331 (1984); (b) 29, 2339 (1984); (c) 29, 2353 (1984).

${ }^{6}$ B. G. Englert and J. Schwinger, Phys. Rev. A 32, 26 (1984); 32, 36 (1984); 32, 47 (1985).

${ }^{7}$ See, e.g., N. H. March, in Theory of the Inhomogeneous Electron Gas, edited by S. Lundqvist and N. H. March (Plenum, New York, 1983), pp. 1-78.

${ }^{8}$ Density Functional Methods in Physics, edited by R. M. Dreizler and João da Providencia (Plenum, New York, 1985).
${ }^{9}$ E. Santos and A. Villagrá, Phys. Rev. B 6, 3135 (1972).

${ }^{10}$ D. P. DiVincenzo, E. J. Mele, and N. A. W. Holzwarth, Phys. Rev. B 27, 2458 (1983).

${ }^{11}$ E. Gaite, M. Leal, and E. Santos, Phys. Rev. B 31, 8226 (1985).

${ }^{12}$ E. Santos and M. Leal, Phys. Rev. A 28, 459 (1983).

13J. Rose, J. R. Smith, and J. Ferrante, Phys. Rev. B 28, 1835 (1983); J. L. Graves and R. G. Parr, Phys. Rev. A 31, 1 (1985).

${ }^{14} \mathrm{~W}$. Kohn and P. Vashishta, in Theory of the Inhomogeneous Electron Gas, Ref. 7, pp. 79-147.

${ }^{15}$ D. C. Langreth and M. J. Mehl, Phys. Rev. Lett. 47, 446 (1981); Phys. Rev. B 28, 1809 (1983).

${ }^{16}$ C. H. Hodges, Can. J. Phys. 51, 1428 (1973). 